\title{
ZUR INTERMEDIALITÄT DES TIERES. THE BAA-LAMB'S HOLIDAY
}

\author{
JÖRG WIESEL
}

Eine Musik geht nicht mehr aus dem Kopf, die die Inszenierung, die hier analysiert werden soll, rhythmisiert und begleitet, ja, unterbrochen hat. Es handelt sich um George Delerues Musik zu Jean-Luc Godards Film LE MÉPRIS (DIE VERACHTUNG) aus dem Jahr 1963. Und bei der Inszenierung handelt es sich um DAS FEST DES LAMMS $^{1}$, das 1940 entstandene Drama (THE BAA-LAMB's HolidAY) der 1917 in Lancashire, England, geborenen Leonora Carrington. Frank Baumbauer, der Intendant der Münchner Kammerspiele, trug dieses Stück seit Jahren mit sich herum. Erst in München, zu Beginn von Baumbauers Kammerspiel-Intendanz (in der zweiten Spielzeit), bot sich eine konkrete Inszenierung an. Premiere war am 30. April 2003: mit dem Regisseur Jossi Wieler und dem Dramaturgen Tilman Raabke, dessen Münchner Textfassung jetzt vorliegt. Das Stück ist spätestens in seiner französischen Übersetzung als LA FÊTE DE L'AGNEAU und seit seiner Uraufführung als Musiktheater unter dem Titel BÄHLAMMS FEST nicht mehr unbekannt. Der österreichischen Komponistin Olga Neuwirth gelang 1999 der Durchbruch mit der Textvorlage Carringtons, das Libretto stammte von Elfriede Jelinek. Und ohne diese musiktheatrale Umsetzung als BÄHLAMMS FEST sind Inszenierung und Dramaturgie der Münchner Kammerspiele - so meine These - nicht zu denken.

Die Geschichte, die das Drama, die Oper und die Inszenierung erzählen, ist so kompliziert wie einfach: Während des Weihnachtsfestes, dem Fest des Lamms, tragen sich im Haus der matriarchal dominanten Mrs Margret Carnis merkwürdige Dinge zu. Das Haus, dessen Beschreibung in der Münchner Inszenierung dem Diener Robert (gespielt von Matthias Bundschuh) in den Mund gelegt wird, ist eine gespenstische Herberge, ein unheimliches Heim lebender Toter, ein Hotel für sexuell unruhige Figuren, deren familiärer Zusammenhalt einzig durch die Autorität der Mutter (Hildegard Schmahl) beglaubigt wird, deren Name programmatisch die Fleischlichkeit ihres Körpers und die Lust zur Überschreitung desselben markiert. Die zweite Ehe ihres alkoholkranken ältesten Sohnes Philip mit Theodora kriselt seit langem und droht am Ekel und der sich zunehmend entwickelnden Verachtung der jungen Frau zu zerbrechen:

»Dieses ganze Haus stinkt nach Alter. Nach Alter und Tod. Laß mich in Frieden. Ich krieche dir nicht nach. Warum kannst du mich nicht einen Augenblick in Ruhe lassen? Ich tue niemandem etwas Böses, nicht einmal deiner ekelhaften, verfressenen Mutter « (Münchner Kammerspiele 2002/03a: 6).

Theodoras Wunsch nach einem emphatisch besetzten anderen Leben, das nicht nur sexuell intensiver als das Leben in ihrer Ehe sein soll, findet sie in der Begegnung

1 Regie: Jossi Wieler, Bühne: Jens Kilian, Kostüme: Gesine Völlm. 
mit Jeremy, dem jüngeren Bruder Philips und - der Text legt diese Spur auf jeden Fall aus - Sohn einer Beziehung zwischen Mrs Carnis und ihrem Lieblingshund Henry (Walter Hess).

Jelineks Libretto szenographiert Henry in einem Setting, das die Reflexion von (Musik-)Theater und Film und Morphing ausbuchstabiert: Jeremys (gespielt in München von Hannes Hellmann) diabolischer Auftritt im zweiten Akt ist der eines wilden Tieres, einer Figur zwischen Mensch und Tier, eines Werwolfs, dem die Spaltung in Mensch und Tier als Zeichen seiner ungeheuerlichen Zeugung immer schon eingezeichnet ist. In dieser surrealen »zoo-politischen Metamorphose « hat Jeremy als Sohn eines Hundes und einer Frau das

»Wölfische am Wolf, das undankbare oder diskriminierende Schicksal übernommen, jene Grenze zu ziehen, die nicht das Tier vom Menschen, sondern eben das menschliche Leben vom menschlichen Leben, den Bürger vom Bürger trennt« (Matala de Mazza/Vogl: 285).

Neben der deutlichen Rezeption surrealistischer Paradigmen wie Salvador Dalís/ Luis Buñuels UN CHIEN ANDALU (1928), der bereits mit Schnitten zwischen Mensch und Tier, Mädchen und Werwolf arbeitete ${ }^{2}$, bewegen sich Carringtons Text und Neuwirths/Jelineks Musiktheater in einer Geschichte von »Verwandlungen, von Metamorphosen, [...] mit denen sich die Politik die Grenze des Politischen und die Menschen-Gesellschaft die Grenze des Geselligen erzählt.« (Matala de Mazza/ Vogl: 286). Jeremy ist als hybrides Geschöpf »an den Rändern des homogenen Kollektivkörpers « (ebd.: 292) situiert; auf ihn fällt am Weihnachtsabend der Verdacht, wenn der Schafhirte Joe Green zuerst ein getötetes, gerissenes Lamm hereinbringt, dann selbst in Gegenwart der Familie tot umfällt.

Jeremy ist bei Carrington die zwitterhafte Bestie mit enorm erotischer Ausstrahlung, seine Animalität ist das Abjekte einer bürgerlichen Genealogie und Gemeinschaft:

»Das Ungeheuerliche der Mischwesen lässt sich darum nicht auf eine Akkumulation verschiedenartiger Bestimmungen zurückführen, auf eine Monstrosität, die aus der Überdeterminierung des Tierhaften am Tier erwächst. Das Unheimliche, Ungeheuerliche der Menschenwölfe geht vielmehr aus dem Verlust symbolischer Markierungen hervor, von Markierungen, die zugleich die Grenze aller Demarkierungen setzen. Es ist die Defiguration in der Figur, es ist der Grund oder Abgrund, vor dem sich die Menschen-Form selbst abzeichnet - ein aufsteigendes Tier, eine aufsteigende Tierheit oder Bestialität« (Matala de Mazza/Vogl: 292f).

So ist die Geschichte des Textes und seiner Inszenierung scheinbar leicht erzählt; sie lässt aber eine ganz zentrale Ebene des Dramentextes unerwähnt: die bei Leonora Carrington surrealistisch in die 1. Szene des dritten Aktes montierte Weihnachtssequenz eines erotisch aufgeladenen Tanzes der Schafe. Während dieses Tanzes erscheint Jeremy mit Theodora. Jeremy ist als Erzengel Gabriel, Theodora als eine »mindere Heilige« (Münchner Kammerspiele 2002/03b: 16) kostümiert. Im Dramentext tötet Jeremy das Schaf Mary, aus dem Tilman Raabke in seiner Münchner Fassung die Figur des Dienstmädchens Violet gemacht hat. Der surreal anmutende Einbau sprechender Tiere in der rhetorischen Figur der Prosopopoiia ist eine Sequenz, die das klassische Theater sprengt, es an seine Grenzen der Reprä-

2 Dazu ausführlich Reck (1994: 261-270). 
sentation treibt, es anhält, stillstellt und unterbricht. Jeremy, der Mörder, wird in der letzten Szene der Münchner Inszenierung von Elizabeth, Philips erster Frau, erschossen und erscheint Theodora als Gespenst.

In der Oper bedient sich Neuwirth für die Darstellung von Jeremy, der hier immer in der Meute, im Rudel von Wölfen auftritt (vgl. Deleuze/Guattari: 326ff), eines Verfahrens von Klangmorphing, es war ihr Wunsch, das klangliche Verschneiden von Mensch und Wolf live zu generieren. Am Grazer Institut für Elektronische Musik hat sie das Timbre kanadischer Wölfe analysiert und herausgefunden, dass sie der Stimmlage eines Countertenors entsprechen. »Dazu musste die Klanganalyse des Countertenors [Jeremy: Andrew Watts, J.W.] im Moment des Singens stattfinden und gleichzeitig der interpolierte Klang resynthetisiert werden « (Höldrich: 42). ${ }^{3}$

Es ist jetzt wichtig für meine Argumentation, dass der im Drama Carringtons angelegte Prozess des Tier-Werdens im intermedialen Einsatz eines anderen Mediums - des Films - in der Münchner Inszenierung aufgefangen, wiederholt und gedoppelt wird. Genauer: Der anfangs erwähnte Film Jean-Luc Godards, LE MÉPRIS, der auf dem Roman IL DISPREZZO (1954) von Alberto Moravia basiert, wird allein über seine Tonspur in die Inszenierung von Jossi Wieler integriert. Jeremys erster Auftritt im zweiten Akt der Münchner Inszenierung artikuliert sich in der Textfassung wie folgt:

»DIE STIMMEN (Jeremy spricht synchron.) I reread the Odyssee last night ... I finally found something that I've been looking for for a long, long time. Something that is just as indispensible for the movies as in real life: poetry « (Münchner Kammerspiele 2002/03a: 21).

Die Untermalung der Szene mit Musik von Georges Delerue, das blaue Licht im Hintergrund und der gelbe Bademantel Theodoras referieren ganz klar auf Godards Film. Der ein zweites Mal in der Textfassung zitierte, in der Inszenierung wieder gestrichene Satz ist einer zentralen Sequenz aus Godards LE MÉPRIS entnommen. Dieser Film erzählt vom Plan eines Filmteams, das in Cinecittà und auf Capri Homers ODYSSEE (ver-)filmen möchte, Regisseur ist Fritz Lang. Paul Naval (Michel Piccoli), ein junger Theaterautor, arbeitet an einem Drehbuch, mit dem der amerikanische Produzent Jeremy Prokosch (Jack Palance) in zentralen Punkten nicht einverstanden ist; es ist ihm zum Teil zu intellektuell. Paul versucht unauffällig, Jeremy seine Frau Camille (Brigitte Bardot) anzubieten; er lässt sie in dessen Auto fahren, drängt sie ihm geradezu auf. Camille reagiert auf diesen Plan irritiert,

3 »Für BÄHLAMMS FEST wurde als solche Klangrepräsentation die sogenannte >Additive Resynthese< gewählt. Dabei versucht man, einen Klang als Überlagerung von Teiltönen [...] mit zeitlich veränderlicher Tonhöhe und Lautstärke zu erzeugen. In einer ersten Analysestufe werden die zeitlichen Verläufe dieser Teiltöne so bestimmt, wie sie den Klang am besten beschreiben. Mit den gefundenen Tonhöhen- und Lautstärkenparametern wird eine Gruppe von Sinusgeneratoren gesteuert, deren Ausgangssignale für jeden Zeitpunkt addiert werden und so einen vom analysierten Original ununterscheidbaren Resyntheseklang ergeben. Sind solche Parameter für Ausgangs- und Zielklang bestimmt, lässt sich Morphing dadurch bewerkstelligen, dass bei der Resynthese zwischen den Teiltonparametern der beiden Klänge interpoliert wird. Beim Morphing von Countertenor zu Wolf würden zum Beispiel die Sinusgeneratoren am Beginn durch die Gesangsparameter gesteuert werden und im Verlauf immer stärker die Tonhöhen- und Lautstärkeverläufe des Wolfsheulen wiedergeben.« (Höldrich: 41). 
verwirrt und zunehmend mit Verachtung. In dieser Sequenz begeben sich die vier Hauptfiguren des Films in ein römisches Filmtheater, in dem Prokosch das Casting für die Rolle der Nausikaa in der zu filmenden ODYSSEE organisiert hat. Godard unterbricht diesen Blick der vier Protagonisten (eine frontale Kameraeinstellung) auf die Leinwand ein erstes Mal durch den Kameraschuss eines Photographen sowohl auf die Kamera Godards im off, wie auch den Blick auf uns als Zuschauer auf der metadiegetischen Ebene. Die Position der Kamera wechselt um $180^{\circ}$, begleitet vom Blitzlicht der Photokamera und der Unterbrechung/Stillstellung der Casting-Musik. Camille und Paul nehmen Platz zwischen Fritz Lang und Jack Palance (Jeremy), aber getrennt durch den Gang. Das mehrsprachig geführte Gespräch unterbricht Godard permanent durch die Arbeit der Übersetzerin (Georgia Moll). In diesem Kontext fällt der Satz Jeremys, den die Münchner Fassung integriert: Das Zwischen-Wesen bei Carrington und Wieler zitiert das Interdiktum des Produzenten aus Godards Film. Dessen Vorstellung von poetry ist während des Gesprächs der vier Akteure - gefilmt als Parallelfahrt - zu sehen und zu hören. Die dramaturgische Konzeption der Inszenierung bezieht sich an dieser zentralen Stelle auf eine Sequenz, die bereits in sich mehrfach gespalten ist und Intermedialität in höchstem Maße reflektiert.

$»$ Vor diesem Hintergrund stellt sich für jeden Film Godards die Frage, welche Funktionen darin als Kategorien und reflexive Genres fungieren. Im einfachsten Fall sind es die ästhetischen Genres: Epos, Theater, Roman, Tanz und das Kino selbst. Zum Kino gehört ebenso eine Reflexion über sich selbst wie über die anderen Gattungen, insofern die visuellen Bilder nicht bloß auf unabhängig von ihnen bestehende Tänze, Romane, Theaterstücke oder Filme verweisen, sondern sich selbst anschicken, für die Dauer einer Serie oder einer Episode Kino, Tanz, Roman, Theater zu >machen« (Deleuze: 241).

Im Film Godards zitiert Fritz Lang in seinem Gespräch mit Paul (Piccoli) über die Realität der Welt der ODYSSEE eine Strophe der 1942 entstandenen HollywOODELEGIEN Bertolt Brechts und intendiert mit Horkheimer und Adorno eine Verfilmung der ODYSSEE, wo der Mythos bereits Aufklärung ist und die Aufklärung in Mythologie zurückschlägt.

\section{»JEDEN MORGEN, MEIN BROT ZU VEDIENEN}

Fahre ich zum Markt, wo Lügen gekauft werden.

Hoffnungsvoll

Reihe ich mich ein unter die Verkäufer.«(Brecht: 115)

Der Film stellt Lang aus, der Brecht in der Zusammenarbeit zu HANGMEN ALSO DIE (1942) mehrfach desavouiert hatte, indem er bestimmte, ihn nicht allein für das Drehbuch verantwortlich zeichnen zu lassen. Godard aber, und mit ihm die Nouvelle Vague, reflektieren die filmkritische Position der Dialektik der Aufklärung (1947): Die Faszination für das Hollywoodkino der fünfziger und frühen sechziger Jahre (John Ford, Howard Hawks, Alfred Hitchcock) wird in diesen Reflexionen einer kinematographischen Autorschaft übernommen und im intermedialen Vergleich deutlich akzentuiert.

Zurück zu Jeremy: Die Münchner Inszenierung, die den Soundtrack des Films als rhythmisierendes Intermedium integriert, dabei aber die Bildspur Godards nur auf der imaginären Ebene mitlaufen lässt, sucht ihre Theaterbilder mit der Endlosschleife Delerues zu amalgamieren. Ton und Bild verschmelzen im Affekt des Be- 
trachters. In dieser intermedialen Dramaturgie liegt aber das Potential einer Reflexion über Intermedialität, wie auch die Möglichkeit, das Theater mit sich selbst zu unterbrechen. Im Konzept der Münchner Dramaturgie liegt der Ansatz, dem sperrigen, surrealen Text Leonora Carringtons mit intermedialen Einsätzen zu begegnen.

Die Interrogation des je einen Mediums mit den Mitteln des jeweils anderen bleibt aber auf halber Strecke stehen, was zum einen dem immensen Erwartungsdruck auf die zweite Spielzeit Frank Baumbauers an den Münchner Kammerspielen und zum anderen der Schönheit des Films Godards geschuldet ist, der die Inszenierung erliegt. Auf dem Spiel aber steht eine medientheoretische Position, die Intermedialität als - politische - Chance der Unterbrechung, der Stillstellung, des Aussetzens von Entscheidung versteht:

»Medien übertragen Botschaften, Sichtweisen, Ästhetiken, sind aber definitionsgemäß als Bote und Bedeutungsträger - nicht die Botschaft selbst. Diese Eigenschaft der Medien, nämlich nur Botschaften, Informationen und Daten zu übertragen, ohne ihren Sinn zu beeinflussen, markiert den Ansatzpunkt einer Metaphorologie der Medien, die in diesem Sinnvorbehalt und Sinnaufschub der Medien eine grundlegende Bestimmung von Medialität als Mit-Teilbarkeit zu begründen versucht. Die permissive Durchlässigkeit oder Disponibilität der Medien, mit der wir im Zeitalter der digitalen Simulation konfrontiert sind, ist jenes seltsame Vermögen, vorgegebene Bedeutungshorizonte zu eröffnen, zu verschieben und zu unterbrechen. Die Medialität der Medien konturiert den Horizont, in dem sie selbst nicht >aufgehen< kann: Medien sind indifferent gegenüber dem, was sie speichern, übertragen und verarbeiten. Eben diese Gleichgültigkeit oder Indifferenz gegenüber dem Sinn der Botschaft ist vielleicht auch als In-Differenz lesbar, d. h. als Dazwischenkunft der uns teilenden und dadurch verbindenden Medien, mithin als das, was uns vorausgeht bzw. den anthropologischen Fixpunkt dieses >Uns< oder >Wir< dezentriert« (Tholen: 8f).

Dass Raabkes und Wielers Konzept so prägnant ist, dann aber nicht klar weitergeführt wurde, zeigt die Figur Jeremy in der Münchner Inszenierung exemplarisch an: seine Interposition als die zwischen Mensch und Tier wird im intermedialen Einsatz gedoppelt und unterbrochen. Die bei Carrington angelegte surreale Intermedialität des Menschen selbst, als Spaltung in Mensch und Tier (als Figur einer begehrenden Bestie), ihre - mit Tholen als »anthropologischer Fixpunkt« eingeschriebene intrahumane Trennung, zeigt auf Giorgio Agambens Überlegungen zu Mensch und Tier. Der intermediale Einsatz der Tonspur des Films von Jean-Luc Godard in der Münchner Inszenierung genau an der Stelle, wo der Mensch Tier und das Tier Mensch ist, überzieht die politische Brisanz des Dramas von Carrington mit der Tonspur von beider Verschmelzung. Die Radikalität aber, die sowohl der Text wie auch Godards Film besitzen, öffnet uns für die Überlegungen Agambens in ganz besonderer Weise:

»Aber wenn das zutrifft, wenn die Zäsur zwischen Mensch und Tier in erster Linie das Innere des Menschen durchzieht, dann muss die Frage nach dem Menschen - und dem »Humanismus « - als solche neu gestellt werden. In unserer Kultur ist der Mensch immer als Trennung und Vereinigung eines Körpers und einer Seele gedacht worden, eines Lebewesens und eines lógos, eines natürlichen (oder tierischen) und eines übernatürlichen, sozialen oder göttlichen Elements. Wir müssen hingegen lernen, den Menschen als Er- 
gebnis der Entkoppelung dieser zwei Elemente zu denken und nicht das metaphysische Geheimnis der Vereinigung, sondern das praktische und politische der Trennung zu erforschen. Was ist der Mensch, wenn er stets der Ort - und zugleich das Ergebnis - von unablässigen Teilungen und Zäsuren ist? Diese Teilungen zu untersuchen, sich zu fragen, auf welche Weise der Mensch - im Menschen - vom Nichtmenschen und das Animalische vom Humanen abgetrennt worden ist, drängt mehr, als zu den großen Fragen, den sogenannten menschlichen Werten und Menschenrechten, Stellung zu beziehen« (Agamben: 26).

Jeremys Hybridität ist in der Inszenierung eine doppelte:

»Hybride oder intermediale Zwischenräume sind also mehr und anderes als die unbezweifelbar zunehmende Konvergenz der Medien, welche sich der digitalen Integration vormals getrennter Medien in einem Medienverbund verdankt « (Tholen: 197f).

Mit Georg Christoph Tholen nehme ich »beide Bedeutungen des Hybriden beim Wort, nämlich Mischung und Maßlosigkeit « (Tholen: 198) und begreife die Intermedialität des Tieres als einen Punkt der theatralen und theoretischen Untersuchung von Animalität jenseits einer »Humanität, die das Mandat der integralen Verwaltung der eigenen Animalität übernommen hat« (Agamben: 86), indem sie über das Menschsein im Ausschluss des Tierseins entschied und entscheidet. Und es wären diese politischen und ästhetischen (theatralen) Grenzziehungen zu erforschen und zu diskutieren - nicht nur im Fest des Lamms -, weil es eben keine »unteilbare Grenze zwischen dem MENSCHEN und dem TIER « (Derrida/Roudinesco: 115) geben kann.

\section{Literatur}

Agamben, Giorgio (2003): Das Offene. Der Mensch und das Tier. Aus dem Italienischen von Davide Giuriato. Frankfurt/M.: Suhrkamp.

Brecht, Bertolt (1988): Gedichte 2. Sammlungen 1938-1956 (= Bertolt Brecht. Werke. Große kommentierte Berliner und Frankfurter Ausgabe. Hg. v. Werner Hecht, Jan Knopf, Werner Mittenzwei, Klaus-Detlef Müller, Bd. 12). Berlin/ Weimar: Aufbau; Frankfurt/M.: Suhrkamp.

Chion, Michel (1990): L'Audio-vision. Son et image au cinéma. Paris: Éditions Nathan.

Deleuze, Gilles (1997): Das Zeit-Bild. Kino 2. Übersetzt von Klaus Englert. Frankfurt/M.: Suhrkamp.

Deleuze, Gilles/Félix Guattari (1992): Tausend Plateaus. Kapitalismus und Schizophrenie. Aus dem Französischen übersetzt von Gabriele Ricke und Ronald Voullié. Berlin: Merve Verlag.

Derrida, Jacques/Elisabeth Roudinesco (2006): Woraus wird Morgen gemacht sein? Ein Dialog (2. Aufl.). Aus dem Französischen von H.D. Gondek. Stuttgart: Klett-Cotta.

Höldrich, Robert (2003): »Klangmorphing«. In: Olga Neuwirth: Bählamms Fest. CD, Klangforum Wien, Johannes Kalitzke. Wien: Kairos, S. 40-42.

Matala de Mazza, Ethel/Joseph Vogl (2002): »Bürger und Wölfe. Versuch über politische Zoologie«. In: Sylvia Sasse/Stefanie Wenner (Hg.): Kollektivkörper. 
Kunst und Politik von Verbindung. Bielefeld: transcript (= Masse und Medium, 2), S. 285-298.

Münchner Kammerspiele (2002/03a): Leonora Carrington. Das Fest des Lamms. Fassung von Tilman Raabke. München.

Münchner Kammerspiele (2002/03b): Leonora Carrington - >Das Fest des Lamms . Programmheft. München.

Neuwirth, Olga (2003): Bählamms Fest. CD, Klangforum Wien, Johannes Kalitzke. Wien: Kairos.

Reck, Hans Ulrich (1994): »Dunkle Erkundungen eines verstummenden Echos. Natur im surrealistischen Film, Natur des surrealistischen Films: Zu einer beispielhaften Poetik des Sequentiellen«. In: Karin Orchard/Jörg Zimmermann (Hg.): Die Erfindung der Natur: Max Ernst, Paul Klee, Wols und das surreale Universum. Freiburg im Breisgau: Rombach, S. 261-270.

Tholen, Georg Christoph (2002): Die Zäsur der Medien. Kulturphilosophische Kulturen. Frankfurt/M.: Suhrkamp. 
